\title{
ANÁLISIS DE INTELIGENCIA EMOCIONAL EN MENORES DE FAMILIAS MULTIPROBLEMÁTICAS
}

\author{
Gema Céspedes Romero ${ }^{1}$
}

Universidad de Almería

\section{Resumen}

El presente trabajo tiene como objeto conocer la situación a nivel emocional en la que se encuentran los menores miembros de familias multiproblematicas, centrándonos en la inteligencia emocional y los niveles con los que cuentan dichos miembros. Para ello, conoceremos las características de este tipo de familias y el contexto en el que se encuentran en el menor y las consecuencias que les puede provocar a nivel emocional a estos menores.

Para realizar la investigación se utiliza la escala TMMS-24 mediante entrevista directa a los sujetos.

En los resultados se observan diferencias en las puntuaciones de las diferentes dimensiones de inteligencia emocional en funcion del numero de hermanos y de la edad.

Se plantea como limitación el numero de sujetos, proponiendo mejorar la investigación mediante la ampliación de la muestra.

\section{Palabras clave.}

Familias multiproblematicas, menor, inteligencia emocional y nivel emocional.

\begin{abstract}
The purpose of this work is to know the situation at an emotional level in which the minor members of multiproblematic families are, focusing on emotional intelligence and the levels that these members have. For this, we will know the characteristics of this type of family and the context in which they are found in the minor and the consequences that these minors can cause emotionally.

To carry out the research, the TMMS-24 scale is used through direct interview with the subjects.

The results show differences in the scores of the different dimensions of emotional intelligence as a function of the number of siblings and age. The number of subjects is proposed as a limitation, proposing to improve the research by expanding the sample.
\end{abstract}

Key words:

Multiproblematic families, minor, emotional intelligence and emotional level.

la pluralidad de formas que podemos observar en su seno. Por ello, la familia es considerada como una institución básica de desarrollo y socialización sobre todo en la infancia, que cuenta con unas reglas definidas por el propio recorrido familiar. También se estima como un sistema humano en cambio constante que influye continuadamente y de forma recíproca en sus miembros.

Dentro de la gama de familias que existen encontramos "las multiproblematicas" estas se caracterizan por no contar con los suficientes mecanismos necesarios para llevar a cabo sus funciones. La mayoría se encuentran en contextos los cuales se caracterizan por factores de riesgo y de limitación sociocultural grave, que causan marginación. (Gómez, Haz, \& Muñoz, 2007).

Los miembros de estas familias muestran déficit de habilidades sociales que en ciertas circunstancias no les permite superar situaciones dificultando así su integración social afectando a su vez a los menores, los cuales carecen de herramientas intelectuales, culturales o sociales. (Rozas, 1999).

Tenemos que tener en cuenta como bien hemos dicho anteriormente que una de las fuentes primordiales de socialización en la primera etapa de edad para los menores es la familia, la cual es la encargada de la 
formación del menor en valores, emociones y hábitos.

"La familia es importante en nuestro entorno porque brinda seguridad material y emocional a sus miembros, facilitando la satisfacción de las necesidades básicas, como vivienda, alimentación, vestido y afecto.” (Fernandéz Barutell, 2013).

Por todo ello hay que resaltar que a nivel emocional la familia es un pilar importante al igual que el contexto en el que este situado el menor, pues es a través de esta los menores aprenden las competencias emocionales que posteriormente se arraigaran en la personalidad de este. Goleman (1996) afirma que la vida familiar es el espacio donde se aprende a pensar en sí mismo, en los sentimientos de uno mismo y en las respuestas posibles ante ciertas situaciones por ello es muy importante que el menor cuente con unas figuras que actúen como referente para ellos y así poder avanzar en su socialización. Debido a que los niños aprenden de sus progenitores (referentes) su base de conocimientos emocionales al igual que la identificación y regulación emocional (Salovey \& Mayer, 1997).

Cuando hablamos de conocimientos emocionales y gestión de emociones es inevitable hablar sobre la inteligencia emocional. El avance en el estudio psicológico hizo que durante el siglo XX se introdujera dicho concepto, desde entonces han sido muchos los especialistas que han intentado definir la inteligencia emocional algunos de ellos son Thorndike quien el 1920 definía este concepto como la capacidad de entender y manejar a las personas para actuar sabiamente en las relaciones humanas. Como bien hemos dicho la definición de inteligencia emocional ha ido avanzando con el paso de los años hasta ser definida como "la capacidad de percibir con exactitud, valorar y expresar emociones; la capacidad de encontrar y/o generar sentimientos cuando éstos faciliten el pensamiento y la capacidad de comprender y regular las emociones para promover el crecimiento emocional e intelectual" (Salovey \& Mayer, 1997)

Por todo lo expuesto con anterioridad este proyecto tiene como objeto investigar el nivel de inteligencia emocional que poseen los menores que viven en un contexto de vulnerabilidad, exclusión social y los que se encuentran en un contexto sin riesgos sociales. Para posteriormente comparar los resultados, observando que factores y situaciones son los idóneos para alcanzar un buen nivel de inteligencia emocional y cuáles son los más perjudiciales.

\section{MARCO TEÓRICO}

El concepto de familia ha sido abarcado por diferentes ciencias como derecho, psicología, sociología entre otras, cada una ha aportado distintos puntos de vista sobre la definición de familia consiguiendo con esto establecer una determinación de familia compleja. La familia durante toda la historia ha estado limitada y afectada por las distintas normas de la sociedad, la economía y los patrones culturales que existieran en cada clase social y en cada parte del país. Contando también con la influencia del modelo de elaboración que predomine en el país, pues este determina el status que tienen en la sociedad basándose en la organización del trabajo y la inserción en él (Valladares González, 2008).

Por otra parte, los expertos que investigan este tema consideran que la familia es una estructura activa, que siempre está en constante cambio del mismo modo en el que la sociedad progresa, y es el resultado que manifiesta la cultura del sistema social. La unión de sistemas individuales compone una institución denominada familia que a su vez a través de interactuar entre ellos hacen que surjan sistemas abiertos. Esta institución, forma parte del sistema social, dando respuestas a sus normas culturales, a sus avances ético-morales, a sus creencias de carácter religioso o a su ideología política (Valladares González, 2008).

La familia está sujeta a la influencia del entorno que les acordona. Manifestación de ello es que a lo largo de la década de los ochenta la organización y las actividades familiares a desempeñar han padecido cambios para adaptarse a las transformaciones que estaban sucediendo en el país y en otros órganos. Desde este punto de vista, la conservación de la familia ha sido posible debido a su capacidad de acomodarse a los cambios que han acontecido en la sociedad. Como consecuencia de los veloces cambios que se dieron hicieron que se pusiera de manifiesto la firmeza de la estructura familiar. En las últimas dos décadas, ha aumentado la frecuencia y diversidad de diferentes tipos de familias, entre las que podemos encontrar el modelo narcisista, el comunitario, el libertario, el sistémico, el laboral, el post-divorcista entre otros. Pero los cambios sociales también han sacado a la luz el problema que ataca y debilita a la familia tradicional y al matrimonio, como por ejemplo el divorcio o las separaciones. Pero donde se ha dado gran parte de los cambios ha sido en las actitudes y valores (Polaino-Lorente \& Martínez Cano, 2003).

Existen una gran variedad de familias algunas de ellas mencionadas anteriormente, pero en este trabajo no vamos a hablar de ellas para así poder centrarnos en profundidad en las familias multiproblematicas. El concepto de Familia multiproblematicas surgió alrededor de los 50 por personas cualificadas y especialistas de los países anglosajones los cuales realizaban investigaciones, estos expertos eran procedentes de trabajo social. Inicialmente este concepto se centra en las familias con un bajo nivel socioeconómico dejando a un lado las relaciones entre los miembros (Martínez, 2003).

Mazer (1972) detecta que si no se escogen familias teniendo en cuenta la base de las relaciones que estas tienen con los distintos servicios o agencias no se puede verificar con claridad una inclinación en la familia multiproblematicas al encontrarse en ambos extremos del espectro socioeconómico. Otros especialistas han observado que hay familias que se encuentran por encima del umbral de pobreza, las cuales tienen problemas a la hora de administrarse favorablemente esto conlleva a que entren en una espiral cíclica donde alternan fases de bienestar con fases de crisis

Salvador Minuchin (1967), manifiesta en su obra "Family of the Slums" que la actividad de dichas familias a nivel comunicacional, estructural y afectivo. Los datos conseguidos dejan claro para algunos terapeutas que no existe un tipo de estructura familiar concreto de cultura-pobreza, rompiendo así la fábula que unía pobreza con desorganización.

Otros autores que centran el foco de la atención en la organización del grupo y en las diferentes variantes de las relaciones que estas familias tienen con el medio social periférico definen a las familias multiproblematicas tal que así (Cancrini, 1995):

- Familias las cuales resaltan por encontrarse en un contexto aislado (Powel, Monahan, 1969) para destacar la soledad que se encuentra en el centro familiar en el medio de la familia extensa, notándose la ausencia de apoyo en las fases críticas de la vida familiar, indistintamente de la clase social de la que procedan.

- Familias que se encuentran discriminadas (Thierny, 1976) para distinguir las características disfuncionales desde una visión constitutiva, debido a las carencias graves de perseverancia en el crecimiento de las respectivas funciones que desempeñan cada miembro haciendo hincapié a nivel del subsistema parental.

- Familias asociales (Voiland, 1962) para marcar los aspectos que atañan al crecimiento de conductas desviadas del ámbito social.

- Familias desorganizadas según Minuchin (1967) en las cuales se muestran vacios a nivel emocional, de comunicación y afectivo.

Partiendo de las investigaciones anteriormente expuestas, las cuales muestran como en dichas familias hay problemas en el desarrollo de las funciones a desempeñar, concretamente en los roles de los 
progenitores, como en la carente acotación de los sistemas, así como la predisposición al desequilibrio a nivel psicosocial de los individuos en los subsistemas, debido a una variabilidad en la organización estructural y dónde un gran número de miembros muestran o denuncian situaciones problemáticas, se podría decir que una gran parte de las familias merecerán la calificación de multiproblematicas.

En las Familias Multiproblematicas el desarrollo de las conductas sintomáticas se confirma regularmente alrededor de las primeras transformaciones de fase del ciclo vital (creación del vínculo entre dos personas, nacimiento y crecimiento de los niños). Las conductas sintomáticas son eficaces a la hora de bloquear la continuidad a la fase siguiente, pero no consiguen mantener el equilibrio previo, participando de forma importante a la organización, partiendo en cambio de modelo importante a la disposición, a la dispersión del núcleo familiar y dando lugar a una situación marcada por :

- Un mal desempeño del sistema familiar que no es capaz de realizar de una forma favorable sus responsabilidades estructurales (atención de los hijos, desarrollo, seguridad de las personas más vulnerables) y afectivas (gestión de las tensiones, alimentación anímica de los menores, solución a las demandas de intimidad y de equilibrio afectivo en los componentes de la familia).

- Una búsqueda de personas ajenas al sistema (familia) las cuales cuenten con una capacidad de desarrollar dichas tareas, personas que se convierten en primordiales las cuales favorecen al decrecimiento de las funciones de los componentes del sistema familiar.

Por otro lado, las familias multiproblematicas cuentan con una serie de características que podemos agrupar en cuatro grupos los cuales son: nivel social, ciclo vital de la vida familiar, paternidadconyugalidad y comunicación-sistema afectivo.

Con respecto, al nivel social uno de los factores de riesgo más importantes es la miseria, debido a que la ausencia de medios y competencias sociales favorece el surgimiento de comportamientos conflictivos y dificulta una resistencia eficiente ante ellos.

En el Congreso de Terapia Familiar (1996), Marcelo Packman afirmaba que lo más destacado de la pobreza era que te permitía predecir lo que iba a suceder. Son familias en la cuales es más frecuente encontrar algún miembro en paro, el ingreso en la cárcel, la aparición de enfermedades, etc.

La desestructuración a nivel económico y de hogar de este sistema incrementa las consecuencias de la pobreza. Los hogares se encuentran muchas veces en un estado de pobreza y de descuido. En su interior una de las características más frecuente es la ausencia de limitación de los espacios, dando lugar a que niños, adolescentes y jóvenes adultos no cuenten con un espacio propio donde puedan construir su identidad personal, lo que conlleva unas consecuencias sorprendentes sobre la determinación de las funciones y las relaciones. Por ello, la estructura del espacio-tiempo de la vida diaria cuenta con un matiz nefasto.

Minuchin (1967) contempla que estos menores de edad desarrollan una vivienda "no tengo un lugar propio en el mundo" y "el mundo sirve para estimularme". "Yo soy un recipiente pasivo de la estimulación que me lleva aleatoria e imprevisiblemente, a la cual no podré jamás controlar."

La manera con la que consiguen los ingresos económicos a menudo se encuentran fuera de los marcos legales y son utilizados para distintos objetivos, para la mujer su objetivo es destinarlo para fines familiares mientras que para el hombre su objetivo es para objetivos personales, lo que conlleva que la suma de estos objetivos nos lleve a una situación en la cual resulta muy difícil establecer criterios de prioridad; debido a que existen unos objetivos costosos e inútiles, mientras se da una gran ausencia de los bienes de primera necesidad. Hay que tener en cuenta que también hay familias las cuales durante un tiempo formaban parte de una clase social integrada en la sociedad y la pobreza es la consecuencia de una desestructuración arraigada.

En cuanto al ciclo vital de la vida familiar, para este tipo de familias no es de gran importancia la relación entre vínculo que son reconocidos por ley y los vínculos establecidos por afecto, por lo que el conjunto de personas que conviven en un hogar no tiene por qué coincidir con la familia legalmente reconocida.

Fulmer (1989) (Nieto, 1997) equipara las distintas etapas en las que se encuentra la familia a lo largo de la vida de unas familias cuyos miembros son personas cualificadas y familias clase social ascendiente. La decisión a la que llega es que en las familias cuyos miembros con personas cualificadas se produce un prolongamiento de cada una de las etapas de la vida llegando así a dos generaciones mientras que en las otras familias se alcanzan tres generaciones.

Las diferencias en el ciclo vital familiar son que las familias, cuyos miembros cuentan con estudios académicos superiores; el prolongamiento de la adolescencia desemboca problemas que tienen que ver con la autonomía y en las familias pobres la velocidad a la que se aceleran las etapas llevan a una modificación de la estructura (por motivos de defunción, gestión, desocupación de algunos componentes) alimentando el desequilibrio de dicho orden desembocando en la incapacidad de aportación a las necesidades de crecimiento de sus miembros.

Acerca de la parentalidad -conyugalidad, las familias multiproblematicas están dañados los roles de los progenitores, tanto en su faceta nutritiva como en la socializadora. El daño de las primeras dificulta la nutrición de los sentimientos y emociones, por lo tanto no se traslada a los miembros de la familia menores de edad la seguridad firme de sentirse queridos y valorados por sus progenitores. Otra de las causas que encontramos es el desarrollo de un apego muy poco seguro (Cyrunik, 2009) pudiendo desembocar en un aumento de padecer diversos trastornos biopsicosociales (Glaser,

2002).Muchos estudios han mostrado en los niños que han sido maltratados físicamente el dominio de un apego ansioso/ambivalente (Finzi, Ram, HarEven, Shnit, \& Weizman, 2001); por lo tanto los menores que han recibido una combinación de diversas formas de maltrato, abuso y negligencia muestran un estilo de apego desorganizado o ansioso/evitativo (Hughes, 2004). Se debe agregar que las investigaciones en menores que han padecido negligencia o maltrato emocional han mostrado la presencia de varias dificultades consecutivas en la infancia, adolescencia y vida adulta mostrando trastornos emocionales (como baja autoestima, ansiedad o estrés), desconciertos en los comportamientos (como irresponsabilidad y conductas antisociales); agresividad y/o aislamiento social una gran parte de estos niños (Glaser, 2002).

A su vez tenemos que ser conscientes que existen distintos agentes socializadores que son los encargados de difundir normas, valores y modelos de comportamiento. Uno de los más importantes para un menor es la familia, no solo porque es el primer agente con el que cuentan los menores, sino porque es el enlace entre el individuo y la sociedad con el que cuenta. La familia socializa al niño consiguiendo que el menor interiorice los elementos esenciales de la cultura, desarrollen los pilares de su personalidad y la verificación de las perspectivas de los padres, estas última puede coger dos modos (Miller \& otros, 1995):

El primer modo de conformidad se relaciona con la obediencia, haciendo mención a situaciones en las cuales el menor adapta a las demandas o exigencias de sus progenitores con el objetivo de conseguir una recompensa o evadir un castigo. Con este modo se corre el riesgo de que el menor vuelva a su comportamiento inicial una vez que se concluyan las recompensas o sanciones.

El segundo modo de conformidad se designa como el proceso de asimila- 
ción, el cual incluye una trasformación que dura más en el tiempo y autónomo del valor material adjunto a la actitud o la conducta personal.

Así mismo, los niños tienen la capacidad de adecuarse prosocialmente al menos por dos vías: por obediencia o por recompensa/castigo. Es decir, en la obediencia encontramos una estimulación exterior mientras que en la interiorización la estimulación es interior. Por lo que se refiere al último modelo de conformidad hay que decir que es la finalidad de gran parte de las agencias de socialización en concreto de la familia.

En relación con la familia, esta socializa al menor conforme a su modo de vida, que es el resultado de la realidad social, económica e histórica en la que se encuentran. Hay que mencionar además que Diana Baumrind, ha determinado tres tipos de intervención entre hijos y padres pudiendo tener relación con incremento de conductas prosociales: el primer tipo que encontramos es los progenitores dictadores, se caracterizan por ser estrictos e instaurar límites severos en la manifestación de las posibles necesidades del niño. Este tipo de progenitores tienen la expectativa de obtener una respuesta la cual se caracteriza por una obediencia estricta, a su vez estos no dan explicaciones de sus actos; sin embargo utilizan técnicas las cuales afirman su poder (amenazas, castigos o negación de privilegios) para poder obtener la respuesta deseada por ellos en este caso la obediencia. El segundo destaca por esperar de sus hijos una conducta razonable y para ello establecen unas normas claras, pero también aplican reglas y normas mediante órdenes, y castigos cuando creen oportuno establecerlas. A su vez fomentan una comunicación abierta entre ellos y sus menores teniendo en cuenta los derechos de cada uno. Por último, el tercer modo es el de los progenitores permisivos, estos destacan por las escasas de exigencias hacia sus descendientes y por ejercer escasamente un control sobre ellos. Un punto a resaltar es que motivan a que sus hijos expresen y manifiesten sus emociones y sentimientos (Rodríguez Pérez, 2007).

Siguiendo la misma línea y teniendo en cuanta la clase social según G.Villarroel (1990) también podemos diferenciar dos tipos de socialización familiar: entre ellos uno va orientado a la obediencia y el otro a la participación.

La socialización autoritaria se da con mayor probabilidad entre las familias de clases sociales bajas y destacan por el hincapié que empelan los progenitores en la obediencia, el respeto autoritario y los controles externos que tiene que tener sus descendientes. Cuando el niño no cumple las expectativas que el adulto tenía este aplica un castigo llegando en algunas ocasiones a atentar contra la integridad física del menor al igual que la integridad del Yo. Si el menor responde adecuadamente a las demandas del adulto escasas veces es premiado por ello, debido a que el progenitor entiende que está ejerciendo correctamente el rol que le pertenece. Como podemos observar este modelo le da más importancia en aplicar el castigo a la conducta no deseada por el adulto que al premiar las conductas correctas, y en los casos en los que estos se llevan a cabo los menores son premiados con objetos materiales. Esto conlleva a que encontremos una comunicación cerrada en la cual el adulto no dialoga con el menor y solo se limita a ordenar las conductas que él considera adecuadas y las que no. Estas órdenes son transmitidas al menor mediante mensajes verbalizados y no verbalizados (Villarroel, 1990).

Por lo que se refiere a la socialización autoritaria resalta el modelo de estructuración familiar en el cual los miembros alcanzan la cohesión y unión mediante los roles tradicionales los cuales se complementan unos a los otros. En este tipo de roles es muy habitual encontrar al padre desempeñando el rol de abastecedor y la madre el rol de ama de casa, consistiendo la socialización primordialmente en el aprendizaje y el traspaso de conductas tradicionales; consiguiendo así que los demás componentes de la familia sean un modelo para el niño, el cual se esforzara por reproducir sus conductas. Como consecuencia de esto el menor se encontrará en un contexto en el cual las relaciones están establecidas con anterioridad y los roles de cada componente estas definidos siendo imposible modificarlos.
Sin en cambio en la socialización participativa se dan con gran frecuencia en familias de clase social media y alta, y destaca por utilizar como herramienta los premios más que las sanciones, reforzando más las conductas adecuadas que las que consideradas como inadecuadas. Cuando con reforzadas utilizan mecanismos simbólicos en vez de materiales a diferencia del modelo de socialización autoritario. (Rodríguez Pérez, 2007)

Por lo tanto, cuanto más usen los ascendientes el poder los menores menos interiorizaran reglas perdurables al comportamiento prosocial, el uso excesivo de este poder puede convertirse en abuso desembocando con ello la aparición de efectos perjudiciales. Mientras que la calidez y el afecto están demostradas como técnicas eficaces y favorecedoras para el desarrollo del comportamiento prosocial combinadas con otras técnicas de educación infantil. (Miller \& otros, 1995)

En las familias multiproblematicas es muy común el fracaso con respecto a las competencias socializadoras en el cuidado del menor respecto de su entorno saludable y también la normalización o difusión de normas y valores asociados a la cultura, como causa de esto hace que se produzca en los menores un retraso en el crecimiento de la consideración y consideración al entorno social, por lo cual encontramos una relación conflictiva con su entorno (Rodríguez Martínez, 2003).

No solo se ve deteriorada esta parte, pues también los podemos encontrar en la conyugalidad. Muy frecuentemente las parejas son elegidas de forma utilitaria, debido a que ciertas carencias que poseen les llevan a intentar obtener algo en la otra persona que a ellos mismo como hemos dicho les falta, esta situación se da de forma viceversa entre los dos miembros de la pareja. Por lo que hay que decir que es una relación que a nivel de complementariedad nace muerta, lo que da como resultado que los cónyuges se utilicen mutuamente; pero no cuentan con las habilidades necesarias para transmitir sentimientos de afecto y reconocimiento.

Es necesario recalcar que los efectos hacia los menores son de un nivel grave y complejo, es muy frecuente que se den abusos sexuales y maltratos, como causa de que los estímulos violentos circulan sin limitaciones hacia estos, sin el dominio de las funciones protectoras. (Martínez, 2003).

Por lo que se refiere a la transmisión de información y a la estructura afectiva Salvador Minuchin y Colaboradores (1967) confirman que a nivel experimental estos sistemas viven los sucesos como pasajeros, que se dan de forma aleatoria y se dan veloces cambios en las emociones. En los intercambios no se encuentran normas ni cuando ocurren entre adultos y niños por el motivo de que el control disciplinar depende del estado de ánimo que en ese momento tenga el adulto.

Se da una experiencia afectiva en la cual no se observan discriminaciones entre los distintos tipos y matices de sentimientos que se encuentran entre una fase en la cual la respuesta se caracteriza por ser violenta y otra fase afectiva de gran cercanía. Tienen un vocabulario limitado a la hora de manifestar las situaciones sentimentales y las relaciones con otras personas. Lo que los lleva a encontrar una frialdad con respecto a las emociones y favorece no poder diferenciar las emociones que sienten cuando se relacionan con los demás. Cabe resaltar la gran dificultad que encuentran a la hora de incluir las experiencias a nivel sentimental o las relaciones con los demás, esto se da debido a la incapacidad de interiorizar las experiencias emocionales por lo que conlleva a que no adquieren ningún tipo de conocimiento de ellas. Las emociones se viven y se manifiestan sin control alguno y de una forma muy intensa.

Como hemos mencionado anteriormente la familia es uno de los agentes en el proceso de socialización, en este tipo de familias este proceso se caracteriza según Martínez (2003): 
Las soluciones que aportan los progenitores a las conductas de sus descendientes no siguen ningún canon pronosticable.

No hay ninguna regla establecida, claras o sobreentendidas, de conductas que pueden ser adquiridas por el individuo.

La autoridad llevada a cabo por los progenitores es básicamente ejecutada intentando inhibir la conducta.

Esto hace que el menor asocie los comportamientos inadecuados o erróneos con el poder o disposición emocional. La ausencia de unos patrones cuyo objetivo sea normalizar las conductas está ligada a la ausencia de instrucciones que pueda adquirir para comportamientos futuros, lo que conlleva que el niño necesite a sus progenitores de una forma continua con el fin de organizar sus transacciones personales.

A lo que respecta a la propiedad de la comunicación, según Minuchin (1967) llega a la conclusión que la comunicación entre hermanos podemos encontrar amenazas, contra-amenazas, comparaciones y en muy pocas ocasiones se refuerzan los aspectos positivos del comportamiento.

Por lo que se refiere al desarrollo de los menores no solo recibirán influencias de sus padres sino de muchas personas entre ellas los amigos, educadores e incluso otros familiares. Pero la relación más elemental a lo largo de la vida de un menor es aquella en la que reciben una gran constancia y confianza, son las que existen con sus padres (como ya ha quedado constancia anteriormente). Estos se preocupan por enseñarles las herramientas necesarias a la hora de los riesgos físicos que puedan sufrir sus hijos pero no se centran en su salud emocional. Hay que tener en cuenta que los niños cuentan con su propio temperamento, los especialistas Alexander Thomas y Stella Chess llevaron a cabo una investigación para ver cuál es el papel del temperamento para ello siguieron el desarrollo de 140 niños desde que nacieron hasta que llegaron a la adolescencia, como conclusión extrajeron que el temperamento es un factor estable del desarrollo. Sin en cambio los estudios también manifiestan que, aunque el temperamento sea un rasgo genético heredado, en algunos momentos se pueden ver alterados a través de la intervención. También han comparado a distintos tipos de padres entre ellos los padres que fomentan adecuadamente a sus hijos tímidos para que se adapten mejor a las circunstancias, como a los progenitores que los protegen a sus hijos retraídos a los cambios, estos estudios revelaron que menores a los que se les fomento la aceptación se liberaron de sus conductas de timidez a los cinco años. (Shapiro, 2002)

Por otro lado, las investigaciones realizadas a madres que cuentan con un apego seguro muestran que éstas responden a la manifestación y vivencia de emociones negativas y positivas. Estas mujeres verbalizan los estados afectivos del niño, consiguiendo con esto que los menores con cuidados sensibles identifiquen toda la gama de emociones. Por ello es razonable meditar que un progenitor sensible a las señales del menor y a las cuales les da una repuesta con un apego seguro, fomentara una alta inteligencia emocional, lo que conlleva a que se dé una adecuada capacidad de regulación emocional, cuya base es la de bajos niveles de estrés, modificación y asimilación de emociones negativas y refuerzo de las emociones positivas (Páez Rovira, Fernández Sedano, Campos Manzano, Zubieta, \& Casullo, 2006). Confirmando estos datos, se ha encontrado que las personas seguras son mejores analizando las emociones faciales de carácter negativas y perciben mejor las emociones positivas que las personas que son ansiosas (Kafetsios, 2004). Esto tiene repercusiones que afectan a nuestra salud, puesto que tenemos que tener claro que los factores emocionales son decisivos en nuestra vida, debido a que han sido identificadas las emociones perturbadoras y las relaciones de carácter tóxico como factores peligrosos que favorecen al surgimiento de algunas enfermedades. Han sido muchas las investigaciones que han dejado claro que los individuos que manejan de modo más consciente y sosegada su vida afectiva disfrutan de una salud mejor que los mencionados anteriormente (Goleman, 1997). Con respecto a los menores Goleman (1997) comenta que los niños de familias pobres sufren con frecuencia "heridas emocionales" las cuales les marca negativamente su existencia.

Para mejorar estas situaciones contamos con la inteligencia emocional ,este término fue utilizado en 1990 por los psicólogos Peter Salovey y John Meyer, en la actualidad este término es definido por La Real Academia Española tal que así : "es la capacidad de percibir y controlar los propios sentimientos y saber interpretar los de los demás", es decir, la capacidad de identificar y comprender nuestras propias emociones es determinante para poder entender las emociones que sienten las demás personas y con ello sentir empatía (Goleman, 2012)

Para poder alcanzar esta capacidad tenemos que contar con: autoconciencia, autogestión, conciencia social y gestión de las relaciones, pues estos cuatro factores componen la inteligencia emocional. Estos factores al igual que las capacidades de inteligencia emocional se muestran cuando nos encontramos en edades tempranas (infancia) y van aumentando conforme a nuestro aprendizaje vital, si algunos de estos fracasan durante el proceso se puede mejorar en cualquier momento (Goleman, 2012). Durante el desarrollo del menor encontramos dos fases:

La primera sería la que encontramos en el cambio de cinco años a siete años en la cual el menor adquiere una mayor capacidad para gestionar y coordinar los impulsos y los esfuerzos imaginativos, así como mejorar su comportamiento.

La segunda fase la encontramos en la etapa de pubertad en la cual la parte de su cerebro que gestiona las emociones y sentimientos padece un "esculpido" lo que le produce que se pierda una gran parte de la capacidad de imaginación.

Existen una multitud de programas que estimulan el desarrollo de los menores entre ellos encontramos el SEL y los programas de aprendizaje socioemocional, este programa de aprendizaje socioemocional está enfocado para ofrecerles a los menores las lecciones que van necesitando a medida que va desarrollándose su cerebro (teniendo en cuenta las dos fases mencionadas anteriormente) y por lo tanto al desarrollo del menor. En un colegio, situado en una zona problemática y con mucha delincuencia, en el cual los alumnos de entre doce y catorce años manifestaban malas conductas tanto dentro como fuera de las aulas tienen un programa de aprendizaje socioemocional, para desempeñar este programa han situado en todas las aulas un cartel, en el cual hay un semáforo con sus correspondientes luces( rojo, amarillo y verde) a través de ellas consiguen que los niños frenen, calmen sus emociones y piensen antes de actuar, también con estos programas consiguieron bajar el nivel de agresividad entre los alumnos del colegio (Goleman, 2012).

Sin embargo, algunos profesionales consideran que los programas enfocados a la prevención familiar deberían de ponerse en marcha desde que el menor es bebé, pues es el hogar el primer lugar para la prevención de los problemas emocionales y de conducta, el principal argumento que se plantea es que son los padres quienes cuentan con mayores posibilidades para bajar los riesgos relacionados a los problemas moderados y los trastornos emocionales de carácter grave. En la actualidad encontramos programas como el SEL en las instituciones públicas que trabajan la gestión de las emociones con los niños con el objetivo de aumentan la inteligencia emocional en ellos, pero estos programas solo se desarrollan en el horario escolar ,como causa de esto encontramos que estos sistemas no pueden modificar la forma de alimentación, regular las conductas hacia el deporte consiguiendo que lo realicen de forma regular ni tampoco controlar los programas de televisión que consumen o el tiempo que pasan frente a la televisión. Solo los padres pueden llevar a cabo decisiones en el día a día las cuales pueden cambiar el destino de un menor. (Shapiro, 2002) 
Sin embargo, Goleman manifiesta su confusión pues él creía que los menores de hoy son víctimas de los avances económicos y tecnológicos. Estos poseen un nivel bajo en las habilidades que componen la inteligencia emocional, debido a que los progenitores emplean más tiempo en los trabajos que las generaciones pasadas. Por el incremento del movimiento laboral los vínculos con la familia extendida se han visto acortados, el motivo lo encontramos en el tiempo libre debido a que ha sufridos cambios en los cuales han llevado a un organización y estructuración excesiva. Tenemos que tener en cuenta que la vía tradicional de transmisión de la inteligencia emocional (IE) tenía lugar en medio de los cambios de la vida cotidiana (la forma de comunicarse con los padres y resto de familiares y en el ajetreo de los juegos) ocasiones que en la actualidad los menores no tienen de la misma forma. El factor tecnológico también tenemos que tenerlo muy presente, pues los niños en la actualidad pasan una gran parte del tiempo delante de las nuevas tecnologías y solos, Goleman creía que esto para la infancia crearía efectos negativos en la capacidad para relacionarse de los menores pero en las últimas décadas los menores parecen incrementar en estos campos. (Goleman, 1997)

A su vez los avances tecnológicos han conseguido que por primera vez en la historia se pueda conocer el funcionamiento que la intricada masa de células tiene cuando pensamos, sentimos, imaginamos o soñamos con exactitud. El conocimiento de esta información neurobiológica nos permite entender la forma en la que los centros emocionales del cerebro nos inducen a estados emocionales como la rabia o el llanto y la forma en la que podemos canalizar para bien o para mal estos estímulos. Todo esto hace que surjan nuevas soluciones para las crisis emocionales colectivas. (Goleman, 1997)

Pero a la hora de hablar sobre inteligencia encontramos un auténtico desafío para quienes tienen una visión estrecha y aseguran que el coeficiente intelectual $(\mathrm{CI})$ es tan solo un dato de carácter genético que no puede modificarse por las experiencias vitales y nuestras vidas se encuentran determinadas por esta aptitud. Mientras que otros autores afirman que las diferencias en el coeficiente intelectual tienen su origen en un conjunto de habilidades a las que se les ha llamado inteligencia emocional, habilidades entre las que encontramos la capacidad de autocontrol, entusiasmo, perseverancia y la habilidad de motivarse así mismo. Estas capacidades pueden ser trasmitidas a los menores ofreciéndoles así la oportunidad de obtener el mejor rendimiento intelectual. Todo esto también se ve afectado por un imperativo moral, debido a que nos encontramos en una época en la cual nuestra sociedad aparentemente se descompone de una manera veloz, en la que el egoísmo, la agresividad y la avaricia destruyen la benevolencia de nuestra vida social. Por ello el motivo de hacer un hincapié importante en la inteligencia emocional, pues esta crea los vínculos entre los sentimientos, el carácter y los estímulos morales. Al mismo tiempo está la evidencia de que las actitudes éticas esenciales que adquirimos en la vida se arraigan en las capacidades emocionales subyacentes. Pero el estímulo es el medio de transporte de la emoción y que la raíz del impulso es un sentimiento que se extiende y cuya finalidad es manifestarse en un acto. Los individuos que se encuentran controlados por estos impulsos sin tener un autocontrol carecen de una falta de moral, pues esta incapacidad de controlarlos compone la base de la voluntad y del carácter. Basándonos en este motivo llegamos a la empatía, es el origen del altruismo, esta es la capacidad con las que cuenta una persona que facilita la compresión de los sentimientos que tienen los demás. Goleman manifiesta que en la sociedad actual existe una gran necesidad de empatía y altruismo. (Goleman, 1997).

\section{ESTUDIO EMPÍRICO}

\section{Hipótesis}

Para la investigación que vamos a realizar, nos planteamos la siguiente hipótesis:

- Los menores que conviven en un entorno familiar multiproblematico presentan niveles de inteligencia emocional general bajos.

- Los menores que conviven en un entorno familiar multiproblematico presentan niveles de más bajos en reparación emocional que en claridad y atención emocional.

\section{Objetivos}

\section{Objetivos generales.}

- Estudiar el estado emocional de los menores que forman parte de una familia multiproblemáticas.

- Analizar el nivel de Inteligencia Emocional que posen los menores que se encuentran en un contexto problemático.

- Inferir si el contexto en el que se encuentra el menor afecta al nivel de inteligencia emocional que posee.

\section{Objetivos específicos.}

- Averiguar la importancia que le dan a sus sentimientos y emociones.

- Conocer si saben gestionar las emociones y sentimientos que tienen.

- Visualizar si saben identificar las emociones de otras personas.

- Descifrar si dedican tiempo a conocer las causas de las emociones y sentimientos.

- Saber si saben identificar sus propias emociones.

\section{Metodología}

El presente trabajo de investigación se ha elaborado siguiendo un enfoque metodológico cuantitativo, utilizando como técnica de recogida de información la implementación de una escala.

En un sentido amplio, la metodología de tipo cuantitativa permite poder medir y analizar los datos obtenidos con una mayor exactitud, debido a que la relación entre una variable independiente y una dependiente se estudia al detalle desembocando en una mayor objetividad con los resultados de la investigación.

\section{Participantes}

Los datos obtenidos de la muestra han sido substraídos de las encuestas realizadas a 30 menores $(100 \%)$ de los cuales 11 han sido hombres y 19 mujeres, es decir, el sexo que predomina en esta muestra es el femenino con un $63.3 \%$ seguido del masculino con un $36.7 \%$ (Fig. 1).

Figura 1

Distribución de la muestra según sexo

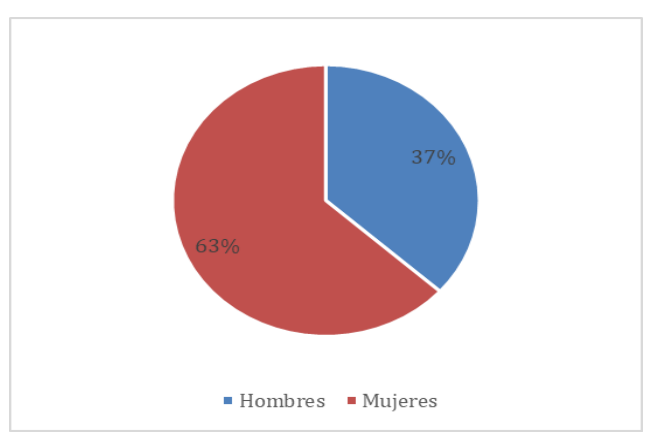


Con respecto a la edad encontramos a 5 menores los cuales se encuentran en la franja de edad de 6 a los 8 años, estos representan al $16.7 \%$ el porcentaje más bajo, en la franja de los 9 a los 11 años encontramos a 9 menores, con un porcentaje del $30 \%$ siendo el porcentaje más elevado, en la franja de 12 a 14 años hay 8 menores con un porcentaje del $26,7 \%$,en la franja de $15-17$ años hay 8 menores representando el $26.7 \%$, en estas dos últimas franjas encontramos que los porcentajes están igualados (Fig. 2).

Figura 2

Distribución de la muestra según edad

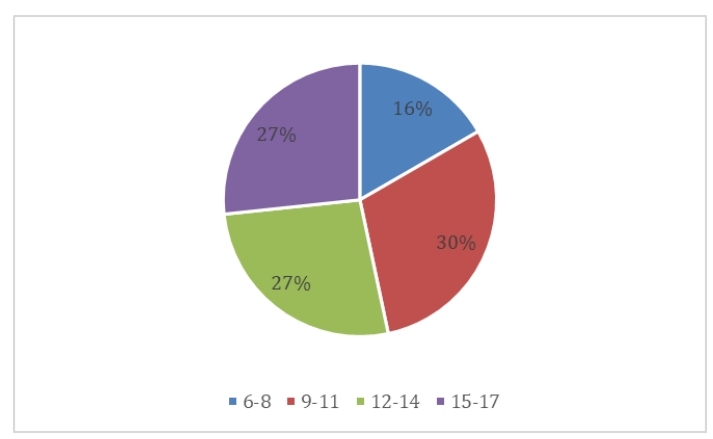

\section{Procedimiento.}

La muestra ha sido tomada a usuarios del Equipo de Tratamiento Familiar (ETF) en estos equipos encontramos con plantilla de profesionales (trabajador social, psicólogo y educador social) los cuales en conjunto trabajan con familias que padecen un importante grado de desestructuración en las cuales se encuentran menores en riesgo social.

Para llevar a cabo esta investigación hemos tenido en cuenta a las personas a las que iba destinada, menores de edad, por ello en todo momento estuvo muy presente la Ley Orgánica 15/1999, de 13 de diciembre, de Protección de Datos de Carácter Personal cuya finalidad es la de legislar los principios de la protección de datos, los derechos de las personas, las disposiciones sectoriales, movimientos internacionales de los datos, la agencia de protección de datos y las infracciones y sanciones. Y la Ley Orgánica 1/1996, de 15 de enero, de protección jurídica del menor, de modificación del Código Civil y la Ley de Enjuiciamiento Civil, en la cual encontramos en el art.4 de dicha ley, reconocido el derecho al honor, a la intimidad y a la propia imagen. Teniendo en cuenta estas leyes y sabiendo que estamos tratando con menores de edad, los cuales cuentan con unos tutores legales responsables de ellos, se solicitó el permiso de estos para que los menores rellenaran el cuestionario, en el mismo omitimos información de carácter personal para así asegurar la intimidad de los menores. intimidad y la tranquilidad de los menores a la hora de realizar la encuesta y del mismo modo no entorpecer el trabajo de los profesionales.

\section{Instrumentos}

La escala utilizada es TMMS-24 complementado con cuatro preguntas sobre la filiación de los sujetos: edad, sexo, número de hermanos y estado civil de los progenitores. La escala TMMS-24 está compuesta por 24 ítems con los que se pretende evaluar la capacidad con la que cuenta un individuo para identificar sus propias emociones, como la habilidad para gestionar sus emociones y sentimientos de forma positiva para uno mismo y para los demás. Esta encuesta cuenta con una estructura en la cual encontramos tres campos, estando estos a su vez compuestos por 8 ítems cada uno, entre ellos podemos encontrar la atención, la claridad y reparación emocional.

Uno de los modelos más destacados es el modelo de inteligencia emocional de Mayer y Salovey (1997), este modelo de habilidades considera que la inteligencia emocional se encuadra entre cuatro habilidades básicas, las cuales son la habilidad de distinguir, reconocer y exponer las emociones con precisión, la capacidad de crear sentimientos que sean positivos para el pensamiento, la habilidad para asimilar emociones y la capacidad de equilibrar las emociones consiguiendo con ello un crecimiento a nivel de inteligencia y emocional (Fernández Berrocal \& Extremera Pacheco, 2005).

Por otro lado, tiene la finalidad de analizar los siguientes factores:

- Percepción emocional. Esta habilidad consiste en detectar y reconocer los sentimientos que tiene uno mismo como los de las personas del alrededor. Para ello es necesario atender y descomponer con exactitud las señales emocionales que se manifiestan facialmente, los movimientos que realizamos a través del cuerpo y el tono de voz que tenemos según nuestro estado de ánimo. En conjunto hace referencia a la capacidad que posee la persona para reconocer adecuadamente las emociones propias a igual que los estados y sensaciones fisiológicas y cognitivas. Esta habilidad tiene como finalidad la discriminación adecuada, la honestidad y sinceridad de las emociones manifestadas por el resto de las personas.

- Asimilación emocional. Abarca la destreza para prestar atención los sentimientos cuando damos respuesta a los problemas o reflexionamos. Esta capacidad se concentra en como el sistema cognitivo se ve afectado por nuestras emociones al igual a la hora de tomar una decisión esta se ve afectada de manera positiva o negativa según nuestro estado de ánimo. Los estados anímicos influyen a la hora de enfocar los problemas cambiando nuestros puntos de vista, llegando a mejorar los razonamientos creativos. En conclusión, nuestras emociones ejercen un efecto positivo sobre la forma en la que procesamos la información y en nuestras reflexiones.

- Compresión emocional. Consiste en descomponer el extenso y complicado catálogo de señales emocionales, etiquetar e identificar en qué nivel se aglomeran los sentimientos. También incluye una capacidad de reconocer y prevenir los efectos que puede tener el estado de ánimo en la actualidad y en futuras conductas. La compresión emocional no solo se trata de esto también abarca la capacidad de reconocer como se mezclan los diferentes estados emocionales causando emociones secundarias, como por ejemplo los celos. Por otro lado, también cuenta la capacidad de interpretar el significado de las emociones complejas, la de identificar los cambios de unos estados emocionales a otros y el surgimiento de sentimientos coincidentes y incoherentes.

- Regulación emocional. Esta habilidad es la más difícil de la inteligencia emocional. Debido a que incluiría la capacidad y la predisposición a encontrarte abierto a los sentimientos de todo tipo, y razonar sobre ellos para así poder desechar o utilizar la información que va adjunta en función de su utilidad. También cuenta con la capacidad para organizar las emociones de uno mismo y las del resto de personas del entorno, prestando más importancia a las emociones positivas y menos a las negativas. Engloba la gestión de nuestro mundo intrapersonal e interpersonal, es decir, la habilidad para normalizar las emociones de las personas, para ello hay que utilizar diferentes estrategias que regulen las emociones que cambian nuestros sentimientos como las del resto de personas. Esto alcanza los procedimientos emocionales más dificultosos, estos consistirían en la normalización de las emociones con el fin de obtener un desarrollo emocional e intelectual.

Por otro lado, las primeras herramientas en progresar que tenían como finalidad evaluar las habilidades de inteligencia emocional fueron las autoinformes, hoy en día siguen siento una herramienta muy utilizada esto es debido a su fácil gestión y velocidad para conseguir unas puntuaciones y a que confía en los procesos introspectivos como una de las maneras más eficaces de investigar las distintas formas de las emociones y afectos de las personas. En muchas de estas herramientas se les pregunta al individuo el grado de conformidad y disconformidad con varias frases relacionadas con su capacidad de identificar, expresar, gestionar entre otras, las respuestas 
que el individuo da forman un índice que se llama "IE percibida" (Salovey, Stroud, Woolery, \& Epel, 2002). Siguiendo esta línea Salovey, Mayer, Goldman, Turvey y Palfai (1995) crearon una medida, el Trait Meta-Mood Scale, intentaba recoger los aspectos de inteligencia emocional intrapersonal de las personas, centrándose en la capacidad de identificar, gestionar, desechar y recomponer los estados emocionales. En España, los investigadores han llevado a cabo una versión más pequeña y cambiada llamada TMMS-24, esta interpretación cuenta con igual forma que la inicial, cuenta con 24 ítems, los cuales calculan tres aspectos los cuales son: dedicación a los sentimientos, claridad emocional y reparación de las emociones (Fernández Berrocal \& Extremera Pacheco, 2005).

Una vez confirmada la autorización de los tutores legales, los propios profesionales del equipo de tratamiento familiar les facilitaron las encuestas a los menores, este método fue llevado a cabo de mutuo acuerdo entre los profesionales y la estudiante del grado de trabajo social para asegurar la

\section{Análisis de datos}

Para el análisis de datos se ha utilizado el paquete estadístico SPSS versión 19. Se han analizado estadísticos descriptivos, $t$ de student y $A N O$ $V A$.

\section{Resultados}

Según el número de hermanos encontramos cuatro apartados, en el primer apartado "ninguno" (ningún hermano) encontramos a 4 menores (13.3\%), en el apartado " 1 hermano" encontramos a 8 menores (26.7\%), en el apartado " 2 hermanos" 9 menores $(30,0 \%)$ tienen dos hermanos, en el apartado "más de 2 hermanos" 9 menores $(30,0 \%)$ tienen más de dos hermanos. Como podemos observar encontramos una igualdad a nivel de porcentajes y de menores en los dos últimos apartados. Una observación que destacar es el porcentaje del aparatado "ninguno" él cuenta con 13.3\%, es decir, solo hay cuatro menores que no tienen ningún hermano.

Tras rellenar las encuestas se ha obtenido unos datos estadísticos descriptivos los cuales están compuestos por uno mínimos, máximos y una media de la atención emocional, la claridad emocional y la reparación emocional. Con respecto a la atención emocional el grupo ha obtenido un mínimo de 14 puntos y un máximo de 38 puntos en la atención emocional con una media del 26,33. En la claridad emocional tienen 10 puntos como mínimo y 36 puntos como máximo con una media de 22,67 y por último en la reparación emocional encontramos un mínimo de 11 puntos y un máximo de 35 puntos con una media de 18,20. Según nos muestran estos datos a pesar de contar con una atención emocional de media del 26.33 y una claridad emocional de media del 22,67 tienen una media del 18,20 en la reparación emocional, es decir, una media baja con respecto a las demás en la reparación emocional.

Una vez analizados estos datos como grupo vamos a analizarlos según sexo. Los hombres (11 menores) con respecto a la atención emocional tienen un mínimo de 14 puntos y un máximo de 36 puntos dando como resultado una media de 25.73 , en la claridad emocional tienen un mínimo de 10 puntos y un máximo de 35 puntos con un media de 23,09 y por último en la reparación emocional manifiestan un mínimo de 12 puntos, un máximo de 33 puntos y una media de 16,55.Las mujeres (19 menores) en atención emocional tienen un mínimo de 18 puntos y un máximo de 38 con una media en total de 26,68 , con respecto a la claridad emocional manifiestan un mínimo de 17 puntos y un máximo de 36 puntos dando como resultado una media de 22,42 y por último en la reparación emocional los datos muestran que tienen un mínimo de 11 puntos y un máximo de 35 puntos con una media de 19.16 .

Como los resultados muestran que los hombres y las mujeres tienen unas medias diferenciadas por un punto en la atención emocional y la claridad emocional en cambio en la reparación emocional se muestra una diferencia más elevada entre sexos, pues mientras que los hombres tienen una media de 16,55 las mujeres tienen una media de 19,16, por lo tanto las mujeres cuentan con una mayor reparación emocional. A nivel individual tanto los hombres como las mujeres cuentan con una alta atención emocio- nal y una baja reparación emocional (la cual resalta más en los hombres como se ha mencionado anteriormente).

Acto seguido realizaremos el mismo paso, pero teniendo en cuenta las franjas de edad de los menores. Los datos obtenidos en la franja de edad entre 6 y 8 años ( 5 menores) manifiestan que los menores que se encuentran en esta edad tienen un mínimo de 18 puntos y un máximo de 38 puntos con una media de 28,00 en atención emocional. En la claridad emocional los datos muestran 19 puntos de mínimo y 30 de máximo dando como resultado una media de 24,80 y por último en la reparación emocional se ha obtenido 15 puntos de mínimo, 29 de máximo y como consecuencia una media de 21,00. Estos menores muestran una gran atención a sus sentimientos y encuentran dificultades para reparar las emociones. En la franja de edad de los 9 a los 11 años ( 9 menores) los datos muestran 14 puntos de mínimo y 36 de máximo con una media de 36 en atención emocional, con respecto a la claridad emocional en el mínimo tienen 18 puntos y en el máximo 35 puntos dando como resultado una media de 23,67 y por último en la reparación emocional en el mínimo hay 11 puntos y en el máximo 33 con una media de 16,22. Como se puede ver según la media estos menores muestran una atención emocional elevado con respecto a la reparación emocional en la cual han obtenido la media más baja. En la franja de edad de 12-14 años (8 menores) en la atención emocional estos menores han obtenido un mínimo de 18 puntos, un máximo de 33 puntos y una media de 26,75. El mínimo que observamos en la claridad emocional es de 16 puntos y un máximo de 36 puntos con una media de 24,25 . Por último en la reparación emocional se manifiesta 14 puntos de mínimo y 35 de máximo dando como resultado una media de 20. Los datos obtenidos manifiestan que en mayor medida prestan atención a sus emociones y en menor medida a la reparación de sus emociones. Por último en la franja de 15 años a 17 años ( 8 menores) los menores han dado un mínimo de 15 puntos y un máximo de 38 y por tanto una media de 25,75 en la atención emocional, sin en cambio en la claridad emocional encontramos un mínimo de 10 puntos y un máximo de 28 puntos y como resultado un media de 18,63 . Por otra parte, en la reparación emocional encontramos un mínimo de 12 puntos y un máximo de 29 puntos dando como resultado una media de 16,88. Como se puede observar muestran una gran atención emocional, en el caso opuesto encontramos la reparación emocional en la cual han obtenido la media más baja.

Observando los datos obtenidos entre las edades podemos ver que los menores que tiene una mejor media en atención emocional son 6-8 años con una media de 28 , seguido encontramos a los menores de $12-$ 14 años con una media de 26,75 y por último encontramos al resto de edades 9-11 años con una media de 25,56 y los 15-17 años con una media de 25,75 , por lo tanto podemos afirmar según estos datos que los menores que los menores que les dan más atención a las emociones son los menores de 6-8 años. Con respecto a la claridad emocional estos datos cambian pues encontramos una semejanza en las medias de los menores de 6-8 años con una media de 24,80 y los menores de 12-14 años con una media de 24,25 , seguido de los menores de entre 911años con una media de 23,67 y por último los menores de 15-17 años con una media de 18.63 , es decir, los menores que tienen más claros sus sentimientos y emociones son los menores de 6-8 y los de 12-14 años y los que menos claro tienen sus emociones son los 15-17 años. Y por último, en la reparación emocional encontramos un nuevo cambio en el orden pues los menores de 6-8 años cuentan con una media de 21 , acto seguido los de 12-14 años con una media de 20 seguido de los menores de 9-11 años y los 15-17 años con una media muy igualada. Estos datos nos manifiestan que los que reparar sus emociones más fácilmente son los menores de 6-8 años y 12-14 años mientras que el resto de las edades tienen más dificultades.

Los datos obtenidos según el número de hermanos son los siguientes:

La atención emocional de los menores (4 menores) que no tienen 
ningún hermano cuenta con un mínimo de 23 puntos y un máximo de 33 puntos dando como resultado una media de 27 , en la claridad emocional encontramos un mínimo de 16 puntos y un máximo de 28 puntos de los cuales se ha obtenido una media de 22,25 y por último en la reparación emocional se ha recabado un mínimo de 11 puntos y un máximo de 33 y con ello una media de 18,25. Como resultado de las medias podemos decir que los menores les prestan mucha atención a sus emociones y cuentan con un nivel bajo para reparar las emociones dañadas.

Los menores que tienen un hermano ( 8 menores) en la atención emocional han dado como resultado 15 puntos de mínimo y 38 de máximo dando como consecuencia una media de 28,13, mientras que en la claridad emocional han obtenido 10 puntos de mínimo y 30 de máximo dando como resultado una media de 22,75 y por último en la reparación emocional en el mínimo tiene 14 puntos y en el máximo 29 puntos y por tanto en la media 20,50. Estos datos ponen de manifiesto que prestan una gran atención a sus emociones y tiene un poco menos de capacidades para reparar las emociones aunque la diferencia entre las medias no es muy elevada tan solo de 8 puntos.

Los menores que han realizado la encuesta y tienen dos hermanos (9 menores) manifiestan en la atención emocional una media de 25,78 con unos mínimos 18 puntos y unos máximos de 33 puntos. Acto seguido en la claridad emocional con un mínimo de 17 puntos y un máximo de 36 tiene una media del 23,78. A lo que respecta a la reparación emocional de mínimo en comparación con el resto de mínimos han obtenido una puntuación de 13 puntos y un máximo 35 puntos dando como resultado una media del 16,44. Estos datos nos permiten poder afirmar que los menores que tienen dos hermanos muestran una gran atención a su emociones debido a la media alta y una mala capacidad para poder reparar las emociones.

Los que tiene más de dos hermanos ( 9 menores) en la atención emocional tiene un mínimo de 14 puntos y un máximo de 38 puntos obteniendo una media de 25 , en la claridad emocional cuentan con 16 puntos en el mínimo y 35 puntos de máximo lo que conlleva a una media de 21,67 y en la reparación emocional tienen un mínimo de 13 puntos y un máximo de 29 puntos dando como resultado una media de 17,89 . Por lo que podemos decir que los menores que tienen más de dos hermanos cuentan con una media alta en la atención emocional (muestran mucha atención a sus emociones) y un media baja en la reparación emocional (encuentran dificultades para reparar sus sentimientos).

Observando los datos de una forma más generalizada podemos ver que los menores que prestan mayor atención a sus emociones son los que tiene un hermano, seguido de los que no tienen hermanos y por último los que menos según la media en comparación con el resto son los que tienen dos hermanos y más de dos hermanos. Si nos fijamos en la claridad emocional observamos que los datos cambian pues los que más tiene claros sus sentimientos son los menores que tienen dos hermanos seguidos de los que no tienen hermanos y los que tan solo tienen un hermano y por último los que menos tienen claro sus sentimientos según la media son los que tiene más de dos hermanos. Mientras que la reparación emocional los menores que tiene dos hermanos son los que menos cuentan con esa capacidad, seguido de los que tienen más de dos hermanos, de ningún hermano y los que cuentan con una gran capacidad para poder reparar sus emociones son los que tan solo tienen un hermano.

Según el estado civil de los padres los datos cambian los menores los cuyos padres están casado ( 9 menores) en claridad emocional han obtenido un mínimo de 23 puntos y un máximo de 33 dando con ello una media de 27 , mientras que en claridad emocional han obtenido un mínimo de 16 puntos y un máximo de 36 con una media de 24,33 y por último en la reparación emocional 13 puntos de mínimo y 35 de máximo dando con ello una media de 19,89. Como se puede observar estos menores cuentan con una media elevada en atención emocional y una media muy baja en comparación con la anterior en reparación emocional con una media de 19,89. En cuanto a los menores de padres divorciados (9 menores) en la atención emocional han obtenido de mínimo 18 puntos y de máximo 38 puntos con una media de 27,89 , mientras que en la claridad emocional la media es más baja, con una media de 22,56 en el mínimo 16 puntos y en el máximo 30. La media más baja la encontramos en la reparación emocional con una media de 16 resultados de un mínimo de 11 puntos y un máximo de 27 puntos. Por otra parte, los hijos de padres viudos (3 hijos) cuentan con unas medias elevadas en la atención emocional y la claridad emocional, pues en la atención emocional tiene una media del 25,67 con un mínimo de 14 puntos y un máximo de 38 puntos, con respecto a la claridad emocional tienen una media de 24 con un mínimo de 19 y un máximo de 28. Por último, en la reparación emocional la media es la más baja con un 18 , resultado que se ha obtenido de un mínimo de 12 puntos y un máximo de 29. Por último, los menores de familias de distintos tipos de estado civil ("otros") siendo 9 menores los que se encuentran en esta situación, la atención emocional tiene un mínimo de 15 puntos y un máximo de 36 dando con ello una media de 24,33 la más alta en este grupo. En la claridad emocional han obtenido una media cuatro décimas más abajo, es decir, una media de 20,67 resultado de un mínimo de 10 puntos y un máximo de 35 puntos. Mientras que la media más baja la encontramos en la reparación emocional con una media de 18,78 con un mínimo de 14 puntos y un máximo de 33 puntos.

Analizando los datos de los distintos grupos según estado civil de los padres ambos coinciden con una media baja en la reparación emocional destacando sobre todos los menores de grupos divorciados con una media de 16, también coinciden con unas medias más elevadas en la atención emocional sobre todo los menores de padres casados y divorciados con una media de 27.

Discusión

Los cambios que ha sufrido la sociedad hasta llegar a la actual han hecho que las relaciones familiares hayan sufrido cambios, a pesar de esto cambios esta institución no ha dejado de ser el origen en el cual los menores adquieren valores y normas culturales y sociales que fomenten el desarrollo del menor. La adquisición de estos valores incluso hábitos que marcan nuestra forma de pensar y nuestro desarrollo social. Por lo tanto, en la familia los menores aprenden los rasgos primarios necesarios para el desarrollo del carácter y la identidad personal que determinaran al menor como persona. A parte de enseñarnos lo anterior en la familia también encontramos el factor emocional, la humanidad no solo ha avanzado en la rama de tecnológica también en la emocional, pues hoy en día los miembros de la sociedad buscan la aceptación del resto de componentes de la sociedad intentado manejar de la mejor manera su existencia sin prejuicios para obtener una buena salud a nivel físico y psicológico. Pero en el libro de Russell (2003) este autor manifiesta que la sociedad de hoy en día es infeliz argumentándolo con distintas situaciones que encajan en la actualidad del hoy en día. Pero también afirma que el secreto de la felicidad se encuentra en el interés que tiene la persona y las relaciones que se caractericen por ser amigables y favorecedoras. Analizando los medios de comunicación podemos que la mayoría del contenido destaca por ser violento o agresivo, es decir, un contenido que manifiesta una ausencia de autocontrol en contexto sociales dañados, especialmente si en ellos se encuentran menores. Un entorno violento no solo lo es por la falta de control de los impulsos dando como consecuencias agresiones físicas, pues el maltrato infantil no solo está compuestos por esto, en el encontramos más términos considerados maltrato como por ejemplo un castigo, una humillación, cuidados negligentes hacia los menores o adolescentes entre otros muchos, estas últimas son algunas de las características de las familias multiproblematicas a la hora de enseñar normas (Rodríguez Martínez, 2003). En la actualidad podemos encontrar situaciones violentas, pues las relaciones sociales hoy en día son muy competitivas en diferentes aspectos de la vida comenzando desde la escuela, esto desemboca que se generen sentimientos negativos para nuestra salud como depresiones, sentimientos de culpabilidad. Pero las situaciones como las mencionadas anteriormente que tienen que afrontar los menores o las 
que ellos crean, no surgen por la ausencia de información sino por la falta de habilidades emocionales y sociales para afrontar y resolver los problemas del día al día (Fernández \& Extremera, 2005).

Algunos autores afirman que han que hacerles comprender a los progenitores la importancia de educar trabajando las emociones, pues estos aportan al menor unos beneficios personales y sociales, consiguiendo con ello que estos sean unas personas con una cualidad inteligente a la hora de sentir. Destacando que el aprendizaje de las emociones inteligentes no se alcanza solo a través de instrucciones verbales también hay que tener en cuenta la práctica, el entrenamiento y su mejoramiento (Fernández-Berrocal \& Extremera, 2002).

Gestionar adecuadamente las emociones favorece la capacidad de identificar lo que se siente y obtener unas decisiones adecuadas. Morgado (2007) asegura que reconocer las emociones que siente uno mismo necesita que uno mismo sea capaz percibirlas como son lo antes posible; de saber de forma rápida la cualidad del estado de ánimo que tenemos en ese momento, de sentir de forma instantánea lo que estamos viviendo es un sentimiento o emoción. Por lo tanto a mayor autoconocimiento de las emociones mayor será la capacidad para controlar la emoción, de analizar y normalizar las causas que conllevan esas emociones, como por ejemplo el emociones tan primarias como el miedo, la rabia, la frustración entre otras, y con ello saber que actitud es más favorable de tomar y como controlar las conductas frente a dicho sentimiento; esta forma de gestionar la comunicación de las emociones y la habilidad de expresar las emociones manifiestan una alta inteligencia emocional. (Uribe Flórez, 2016) Por ello es posible que los datos obtenidos en los menores a los que se les haya realizado la encuesta muestren una alta atención emocional pero una baja claridad emocional y reparación emocional.

Por otra parte, los menores de padres divorciados son los que muestran los datos más bajos en reparación emocional, debido a la situación que viven estos es más probable que manifiesten problemas de la hora de adaptarse. Pues según un estudio estos menores tanto mujeres como hombres durante al año siguiente al divorcio presentan unas cifras más elevadas de problemas, es decir, de agresiones, delincuencia, consumo de drogas en comparación con los menores de familias estructuradas, estos comportamientos, aunque se manifiestan por igual en ambos sexos muestran una tendencia más elevada de seguir de una manera más continuada en el sexo masculino. Por otra parte, la parentificación instrumental y emocional en las menores de sexo femenino conlleva unos niveles elevados de depresión y ansiedad, mientras que por otra parte la de los menores de sexo masculino que viven con los padres los lleva a una situación de mayor depresión. (Cantón Duarte, Cortés Arboleda, \& Justicia Díaz, 2002).

A lo que se refiere a la claridad emocional habitualmente confundimos los términos sensación, emoción y sentimientos, estos conceptos son aplicados para describir el sistema emocional, pero a la mayoría de los humanos les cuesta diferenciar lo que estos términos abarcan por ellos encontramos a personas a las cuales les resulta complicado definir como se sienten o lo que sienten ante una determinada persona al igual que con ciertas situaciones. Los pensamientos afectan a nuestro estado emocional, debido a que este puede variar en función de los pensamientos. Estos últimos se interpretan según las creencias y experiencias adquiridas en la vida, produciendo así una respuesta emocional y con ella una conducta determinada, los pensamientos pueden ser de dos tipos positivos y negativos, está demostrado que los pensamientos positivos ejercen sobre la persona unos efectos positivos y los negativos por el contrario aportan a la persona aspectos negativos produciendo malestar, es decir, ansiedad. La mente se encuentra en un conflicto sin saber actuar con las respuestas adecuadas, haciendo así que los mecanismos de alerta que se encuentran en él se activen produciendo sensaciones físicas y mentales desfavorecedoras para la persona, produciendo un desequilibrio en el comportamiento armonioso del individuo, cambios de hábitos, irritabilidad, sensaciones de tristeza y sobre todo alteraciones psicosomáticas.(Molina, 2017).

\section{Conclusiones}

Como hemos podido observar a lo largo de esta investigación el contexto en el que se encuentran los menores es muy importante pudiendo afectar de forma positiva o negativa a la inteligencia emocional de estos. La familia es una institución la cual ha sufrido diferentes cambios a lo largo de las etapas cambiando su estructura pero lo que hasta ahora no ha cambio es que es un agente socializador para los menores, es la conexión de los menores con la sociedad, esta es la que les enseña los valores culturales y normas sociales del menor y hábitos, la forma de socializara los menores es importante pues como hemos dicho para estos las figuras de los progenitores es la de enseñanza la forma de utilizar las herramientas es fundamental pes puede marcar la identidad social del menor afectando a sus estados emocionales. Por otra parte esto conlleva también que los menores adopten en las edades tempranas las conductas de sus padres como aceptables y las reproduzcan adquiriendo con ello conductas perjudiciales para ellos en un futuro como por ejemplo no tener un autocontrol de las emociones dejándose influenciar por los impulsos más violentos.

En muchas ocasiones según han afirmado diferentes autores las familias no les proporcionan la atención necesaria a la gestión y la identificación de las emociones desaprovechando los beneficios que la inteligencia emocional les aportaría a sus hijos. Pues la inteligencia emocional es la unión como su nombre dice entre la inteligencia y las emociones, como consecuencia de esta unión obtenemos que este término es considerado como la habilidad para razonar las emociones y la capacidad de mejorar las emociones potencialmente y así conseguir guiar los pensamientos. Como hemos visto en este proyecto la capacidad de poder gestionar las cualidades afecta favorablemente a nivel emocional y de salud de las personas, mostrando un nivel inferior de estrés y ansiedad entre otros aspectos negativos para la salud en las personas que saben ejecutar esta capacidad. Por ello, aunque ya existen programas como el SEL, una vez demostrados los grandes beneficios que esto aporta a la persona decir que la sociedad tiene las herramientas en sus manos solo falta ponerlas a funcionar.

\section{Referencias bibliográficas}

Cantón, J., Cortés, M. d., y Justicia, M. D. (2002). Las consecuencias del divorcio en los hijos. Psicopatología Clínica, Legal y Forense, 47-66.

Carbonell, J., Carbonell, M., y González, N. (2012). Las familias en el siglo XXI: Una mirada desde el Derecho. Universidad Autonómica del Estado de México: Elvia Lucía Flores Avalos.

Cyrunik, B. (2009). Los Patitos feos. La resiliencia. Una infancia infeliz no determina la vida. Barcelona: Gedisa.

Shapiro, L. (2002). La salud emocional de los niños. España: EDAF.

Fernández, L. (2013). Intervención con familias y atención amenores en riesgo social. España: McGraw-Hill.

Fernández-Berrocal, P. y Extremera, N. (2005). La inteligencia emocional y la educación de las emociones desde el Modelo de Mayer y Salovey. Revista Interuniversitaria de Formación del Profesorado, 63-93.

Fernández-Berrocal, P., y Extremera, N. (2002). La inteligencia emocional como una habilidad esencial en la escuela. Revista Iberoamericana de Educación, 1-6.

Finzi, R., Ram, A., Har-Even, D., Shnit, D., y Weizman, A. (2001). Attachment styles and aggression in physically abused neglected children. Journal of Youth and Adolescence, 769-786.

García, M., y Giménez, S. I. (2010). La inteligencia emocional y sus principales modelos: propuesta de un modelo integrador. Espiral Cuadernos del Profesorado, 3(6), 43-52.

Glaser, D. (2002). Emotional abuse and neglect (psychological maltreatment). Child Abuse and Neglect, 26 (6-7), 6. https:// doi.org/10.1016/S0145-2134(02)00342-3 
Goleman, D. (2012). El cerebro y la inteligencia emocional: Nuevos descubrimientos. España: Ediciones B.

Goleman, D. (1997). Inteligencia Emocional. España: Círculo de Lectores.

Gómez, E., Haz, A. M., y Muñoz, M. M. (2007). Familias multiproblemáticas y en Riesgo Social: Características e Intervención. PSYKHE, $43-54$

Hughes, D. (2004). An attachment-based treatment of maltreated children and young people. Attachment y Human Development, 263-278.

Kafetsios, K. (2004). Attachment and emotional intelligence abilities across the life course. Personality and Individual Differences, 37 (1), 129 -145. https://doi.org/10.1016/j.paid.2003.08.006

Martínez, M. R. (2003). La familia Multiproblemática y el modelo sistémico. Portularia: Revista de Trabajo Social , 89-115.

Nieto, L. (1997). Familias pobres y multiasisladas. Madrid: Promolibro.

Páez, D., Fernández, I., Campos, M., Zubieta, E., y Casullo, M. M. (2006). Apego seguro, vínculos parentales, clima familiar e Inteligencia Emocional: socialización, regulación y bienestar. Ansiedad y estrés, 319-341.

Polaino-Lorente, A., y Martínez, P. (2003). Evaluación psicológica y psicopatológica de la familia. Guadalajara: EDICIONES RIALP.

Rodríguez, J. C. (2018). Apego e Inteligencia emocional en familias en riesgo social. Beau Bassin, Mauritius: Editorial Académica Española.

Rodríguez, J. y Rodríguez-Góngora, J. (2018). EntendiéndoTE: Programa de habilidades sociales y auto-reconocimiento y control de las emociones. Baue-Bassin, Mauritius. Editorial Académica Españo-

Rodríguez, J. C., Rodríguez-Góngora, J., Pérez-Fuentes, M. C. y Gázquez, J. J. (2013). Análisis de la relación de apego e inteligencia emocional en jóvenes universitarios. En Investigación en el ámbito escolar (pp. 485-491). Granada, España: Editorial GEU.

Rodríguez-Góngora, J., Pérez-Fuentes, M. y Gázquez, J. (2015). Relación entre el Estilo Educativo parental y el nivel de adaptación de menores en riesgo social. European Journal of Investigation in Health, Psychology and Education, 3(3), 301-318.

Rodríguez-Góngora, J (2015). Estilo educativo parental como valor predictivo del nivel de adaptación e Inteligencia Emocional de menores en riesgo social. Facultad de Ciencias de la Educación, Enfermería y Fisioterapia. Departamento de Psicología. Tesis no publicada. Universidad de Almería.

Rodríguez-Góngora, J., Pérez-Fuentes, M. C., Gázquez, J. J. y Rodríguez, J. C. (2013). Propuesta de estudio sobre la influencia del estilo educativo parental en el nivel de adaptación e inteligencia emocional de menores en riesgo social. En Variables Psicológicas y Educativas para la intervención en el ámbito escolar (pp. 369-374). Almería, España: Asoc. Univ. de Educación y Psicología.

Rodríguez, M. (2003). La familia multiproblemáticas y el modelo sistémico. Portularia, 89-115.

Rodríguez, A. (2007). Principales modelos de socialización familiar. Foro de educación, 91-97.

Rozas, G. (1999). Familia y pobreza dura. Revista de Psicología de la Universidad de Chile, 83-94.

Salovey, P., Stroud, L., Woolery, A., y Epel, E. (2002). Perceived emotional intelligence, stress reactivity and symptom: Further explorations using the Trait Meta-Mood Scale. Psychology and Health, 611 $-627$.

Valladares González, A. (2008). La familia. Una mirada desde la Psicología. Medisur, 4-13.

Villarroel, G. (1990). Modos de socialización familiar y sus efectos en el niño. Chile: Estudios Pedagógicos. 
